\title{
Miller-Dieker Syndrome Associated with Congenital Lobar Emphysema
}

\author{
Linda Mahgoub, MD ${ }^{1}$ Khalid Aziz, MBBS, MA, MEd, FRCPC ${ }^{2}$ Dawn Davies, MD, FRCP $(C)^{3}$ \\ Norma Leonard, BSc, MSc, MD, FRCP(C), $\mathrm{FCCMG}^{4}$
}

${ }^{1}$ Department of Pediatrics, The Stollery Children's Hospital, Edmonton, Alberta, Canada

2 Department of Pediatrics, University of Alberta, NICU Royal Alexandra Hospital, Kingsway, Edmonton, Alberta, Canada

${ }^{3}$ Department of Pediatrics, University of Alberta, Edmonton, Alberta, Canada

${ }^{4}$ Department of Medical Genetics, University of Alberta Campus, Edmonton, Alberta, Canada

Am J Perinatol Rep 2014;4:13-16.

\author{
Address for correspondence Norma Leonard, BSc, MSc, MD, FRCP(C), \\ FCCMG, Department of Medical Genetics, University of Alberta \\ Campus, Edmonton, Alberta T6G 2H7, Canada \\ (e-mail: Norma.Leonard@albertahealthservices.ca).
}

\author{
Abstract \\ Keywords \\ - lissencephaly \\ - Miller-Dieker \\ syndrome \\ - congenital lobar \\ emphysema
}

Miller-Dieker syndrome (MDS) is a rare genetic syndrome associated with lissencephaly, developmental delay, and high mortality. We describe a patient who was diagnosed postnatally with both MDS and congenital lobar emphysema. We believe that this is the first reported case of the two conditions presenting in the same patient.
Miller-Dieker syndrome (MDS) is a rare contiguous gene deletion syndrome that is a subtype of the neuronal migration disorders that causes a severe type of lissencephaly. Congenital lobar emphysema (CLE) also known as congenital lobar over inflation, is characterized by progressive hyperexpansion of an upper lobe or the right middle lobe after birth. We report a female patient with MDS with a terminal deletion of chromosome 17p who developed progressive respiratory distress after birth, and was subsequently confirmed to have CLE. To our knowledge, this association has not been previously reported. This raises the possibility that CLE may be associated with genes present at the tip of the short arm of chromosome 17 including the lissencephaly gene (LIS1) at $17 \mathrm{p} 13.3$.

\section{Case Report}

A female infant was born to a 35-year-old gravida 2 para 1 woman following a healthy pregnancy. Antenatal ultrasound scan at 18 weeks did not show significant abnormalities.
However, a subsequent antenatal scan at 32 weeks was done in view of a clinical suspicion of polyhydramnios that was confirmed with an amniotic fluid index of 38 to $39 \mathrm{~cm}$. There was intrauterine growth restriction (IUGR) below the 10th centile, shortened fetal limbs (measuring 3-4 weeks behind the gestational age), absent nasal bone, ventriculomegaly, and poorly visualized cisterna magna. Before further planned investigations that included fetal magnetic resonance imaging (MRI), fetal echocardiogram, and amniocentesis, there was spontaneous labor at 33 weeks gestation with an uncomplicated vaginal delivery.

A female infant, birth weight $1,530 \mathrm{~g}(<10$ th centile), head circumference $30 \mathrm{~cm}$ (10th-50th centile), length $45 \mathrm{~cm}$ ( $<50$ th centile) was born with Apgar scores of 7 and 9 at 1 and 5 minutes, respectively. She had a mild frontal bossing, low-nasal bridge, anteverted nares, and syndactyly between the second and third toes bilaterally. She did not have mandibular changes, furrowing of the brow, or downslant to the palpebral fissure. These may not have been evident because of her underlying prematurity and growth received

November 13, 2013 accepted after revision November 27, 2013

published online

March 28, 2014
Dol http://dx.doi.org/ 10.1055/s-0033-1364192. ISSN 2157-6998.
Copyright (c) 2014 by Thieme Medical Publishers, Inc., 333 Seventh Avenue, New York, NY 10001, USA. Tel: +1(212) 584-4662.
License terms

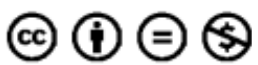


retardation. She had central hypotonia, reduced-spontaneous movements, and a poor cry. Within the first hour of birth, she developed signs of respiratory distress including tachypnea and subcostal recessions, treated with nasal continuous positive airway pressure support (NCPAP).

Head ultrasound showed dilated bilateral ventricles, significant colpocephaly, and bilateral lenticulostriate vasculopathy. Brain MRI revealed absence of gyri with an indentation in the region of the central sulcus, thickening of the cortex with a thin outer layer and a thickened region of the gray matter deep to the cell sparse zone approximately $4 \mathrm{~mm}$ in width, and a normal corpus callosum, cerebellum and brain stem, diagnosing a type 1 lissencephaly (-Fig. 1). A video electroencephalography showed a slowed trace but no epileptiform discharges were detected.

Echocardiogram showed a moderate patent ductus arteriosus (PDA) which was medically treated and an aneurysmal atrial septum. Abdominal ultrasound, ophthalmology examination, and newborn metabolic screen were normal.

Chest radiographs (CXR) showed changes consistent with hyaline membrane disease, with a hyperinflated hyperlucent oligemic left upper lung lobe, and mild atelectasis of the left lower lung. In view of the CXR findings, persistent tachypnea and dependence on NCPAP at 4 weeks of age she had a Tc- $99 \mathrm{~m}$ ventilation-perfusion $(\mathrm{V} / \mathrm{Q})$ scan which showed absent ventilation and reduced perfusion of the hyper expanded upper left lobe. A chest computed tomographic scan confirmed the diagnosis of congenital left lobar emphysema (-Fig. 2).

Chromosomal analysis confirmed an abnormal female karyotype 46,XX,del(17)(p13).ish del (17)(p13.3p13.3)(LIS1-) seen on giemsa-stained banded chromosomes with fluorescence in situ hybridization (FISH) analysis confirming deletion at the

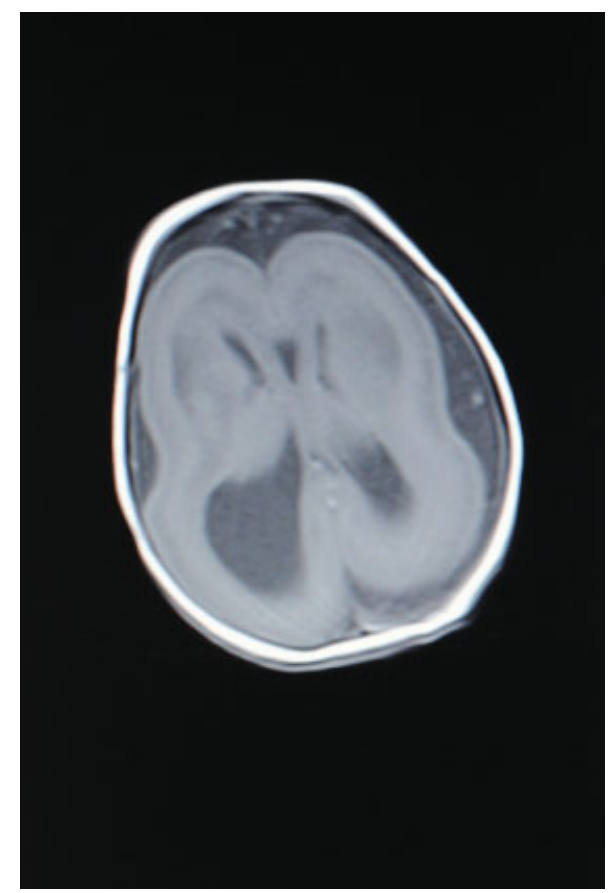

Fig. 1 Brain MRI: T1-weighted axial MRI view of the brain which shows the classical agyria pattern and ventriculomegaly. MRI, magnetic resonance imaging.

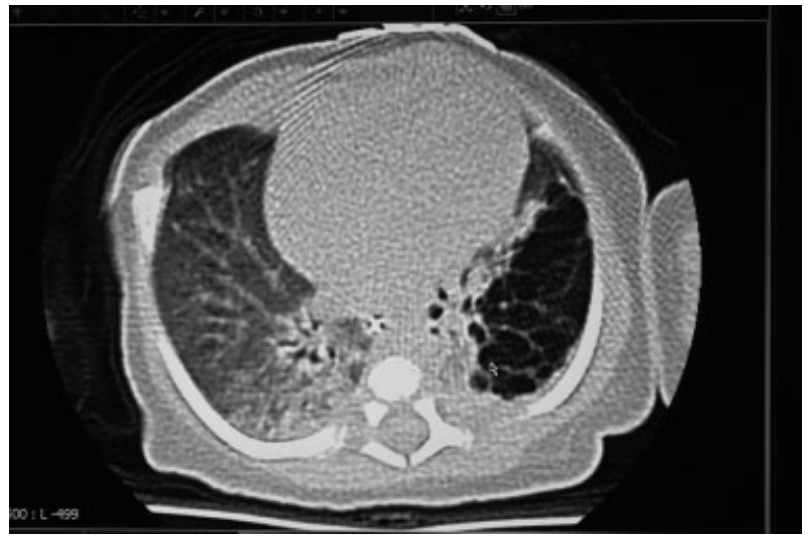

Fig. 2 Chest CT: Unenhanced chest CT image showing a markedly hyperinflated oligemic left upper lobe without cystic or solid component, consistent with congenital lobar emphysema. In addition, there was peribronchial thickening and atelectasis in other lobes with a more confluent consolidation in the right upper lobe due to aspiration pneumonia. CT, computed tomography.

17p13.3 locus (LIS1-), the gene associated with MDS. Interestingly, this patient's deletion was from 17p13.3 all the way to the short arm terminus of chromosome 17 . However, microarraybased comparative genomic hybridization was not available at the time. Parental karyotypes were normal.

The combined diagnoses of MDS and CLE were further complicated by dependency on respiratory support, severe gastroesophageal reflux with recurrent aspiration pneumonia, tube feeding, and failure to thrive. Consequently, a palliative care plan was agreed upon and the baby died peacefully at the age of 70 days.

\section{Discussion}

MDS is a contiguous gene deletion disorder causing type 1 or classic lissencephaly. The gene LIS1 is required for neuronal migration. 17p13.3 haploinsufficiency is displayed by visible or submicroscopic deletions detectable by FISH.

Prenatal ultrasound findings of MDS includes smooth brain, delayed and/or abnormal sulcal development, absent parieto-occipital and calcarine fissures, abnormal sylvian fissure, ventriculomegaly, asymmetric colpocephaly with dilated occipital horns, IUGR, and polyhydramnios. Many of these features may not be present until later in gestation. With the exception of micrognathia, facial dysmorphisms may be too subtle to be appreciated prenatally. ${ }^{1}$ Our patient's third trimester ultrasound findings of ventriculomegaly, IUGR, and polyhydramnios have been reported as important prenatal ultrasound markers of Miller-Dieker lissencephaly syndrome. These should lead to a detailed evaluation of the cerebral sulci via fetal MRI and genetic analysis. ${ }^{2}$ The premature labor seen for our patient is not a known association with MDS, but it may be caused by the underlying severe polyhydramnios.

Postnatal brain image findings of MDS include the typical combination of agyria or pachygyria, four abnormal 
cortical layers, enlarged ventricles, and neuronal heterotopias. ${ }^{3}$ Colpocephaly and incomplete opercularization with wide shallow sylvian fissures are noted to produce a shape similar to the figure $8 .{ }^{4}$ Partial or complete agenesis of the corpus callosum may also be noted. The antenatal and postnatal brain images in our patient were characteristic of lissencephaly in addition to the uncommonly described finding of a thalamostriate vasculopathy. ${ }^{4}$ Lenticulostriate or thalamostriate vasculopathy is detected using cranial ultrasound as hyperechogenic arteries in the thalamus and basal ganglia. It may represent a perinatal diffuse insult to the fetal brain of ill neonates and is not a classic feature of MDS. $^{5}$

MDS is almost invariably associated with early demise, mostly in infancy or early childhood. It consists of severe classical lissencephaly, characteristic facies, hypotonia, joint contractures, seizure activity, severe mental retardation, scoliosis, and failure to thrive. Facial dysmorphism includes narrow and prominent forehead, downward slanting palpebral fissures, small nose, anteverted nares, micrognathia, and a prominent upper lip. ${ }^{6}$ MDS has been associated with other malformations such as congenital heart disease (PDA and ventricular septal defect), genitourinary anomalies (renal anomalies, cryptorchidism, inguinal hernia), gastrointestinal (GI) tract anomalies (duodenal atresia, omphalocele), limbs defects (contractures, polydactyly/syndactyly/clinodactyly), and ear anomalies. ${ }^{7}$

Literature search of MDS did not identify a reported association with any congenital pulmonary disorders. However, a case has been reported of congenital cystic adenomatoid malformation of the lung in association with a large deletion of $17 p$, but MDS was excluded based on the lack of its clinical manifestations and a confirmed intact LIS1 region. ${ }^{8}$ Interestingly, our patient also had a large deletion of $17 \mathrm{p}$ however was at the terminal end, which may include missing genes that predispose to pulmonary manifestations.

CLE is because of over distension of otherwise normal alveoli without destruction of alveolar walls. This may be because of a focal deficiency of bronchial wall cartilage, deficient connective tissue stroma, and intrinsic or extrinsic obstruction of an affected bronchus. Antenatal diagnosis is rare because the hyperinflated lobe is filled with fluid. Most cases present early in life with a progressive respiratory distress. The differential diagnosis includes congenital adenomatoid malformation and bronchogenic cyst. Radiography remains the primary imaging modality. ${ }^{9}$ Treatment of CLE may require surgical resection shortly after birth for severely symptomatic cases, elective surgery within months or in the first years of life for stable symptomatic cases, and conservative management for asymptomatic small lesions. ${ }^{10}$ Elective surgery may prevent chest infections, relieve mass effect symptoms, and eliminate the slight risk of developing a lung mass malignancy. Lobectomy has to be balanced with the risks of the surgery itself and its anesthetic challenges. ${ }^{9-11}$

Associated anomalies with CLE are mostly cardiovascular with other rare organ involvement such as renal, GI, muscu- loskeletal, and cutaneous malformations. ${ }^{12}$ On the basis of our literature search, CLE has not been described in association with congenital brain malformations or MDS. However, studies have identified that deletion at the $17 p$ chromosome as one of the most frequently affected regions in lung cancers, usually occurring as somatic changes often isolated to the tumor tissue alone. ${ }^{13}$ For generalized emphysema, a hereditary component has been suspected to play a role in predisposition for those families with familial emphysema. Emphysema can also be seen in those with severe $\alpha_{1}$-anti trypsin deficiency the gene of which is found on chromosome $14 .^{14}$ To date, there are no clearly identified pulmonary genes found on the short arm of chromosome 17 from 17 p13.3 to the terminus that would cause CLE. One cannot exclude the possibility of other developmental genes in the region that when deleted disrupt normal development of the bronchus or alveoli and lead to CLE.

We conclude that there have not been any reports of MDS, chromosome 17p13 deletions and CLE within the same patient. This case report therefore describes a rare association of two congenital disorders that may occur by chance or due to an unknown underlying pathogenetic link.

\section{Acknowledgments}

We thank the patient's family for their kind permission to publish this report. We declare that there are no conflicts of interest.

\section{References}

1 Fong KW, Ghai S, Toi A, Blaser S, Winsor EJT, Chitayat D. Prenatal ultrasound findings of lissencephaly associated with Miller-Dieker syndrome and comparison with pre- and postnatal magnetic resonance imaging. Ultrasound Obstet Gynecol 2004;24(7): 716-723

2 Lin CY, Chen CP, Liau CL, et al. Prenatal diagnosis of monosomy 17p (17p13.3->pter) associated with polyhydramnios, intrauterine growth restriction, ventriculomegaly, and Miller-Dieker lissencephaly syndrome in a fetus. Taiwan J Obstet Gynecol 2009;48(4): 408-411

3 Wynshaw-Boris A. Lissencephaly and LIS1: insights into the molecular mechanisms of neuronal migration and development. Clin Genet 2007;72(4):296-304

4 Herman TE, Siegel MJ. Miller-Dieker syndrome, type 1 lissencephaly. J Perinatol 2008;28(4):313-315

5 Makhoul IR, Eisenstein I, Sujov P, et al. Neonatal lenticulostriate vasculopathy: further characterisation. Arch Dis Child Fetal Neonatal Ed 2003;88(5):F410-F414

6 Dobyns WB, Das S. LIS1-associated lissencephaly/subcortical band heterotopia. In: Pagon RA, Adam MP, Bird TD, et al., eds. Seattle, WAUniversity of Washington 1993-2013. Available at: http:// www.ncbi.nlm.nih.gov/books/NBK5189. Accessed January 1, 2013

7 Chen CP, Chien SC. Prenatal Sonographic Features of Miller-Dieker Syndrome. J Med Ultrasound 2010;18(4):147-152

8 YamamotoT, Ueda H, Kawataki M, et al. A large interstitial deletion of $17 \mathrm{p} 13.1 \mathrm{p} 11.2$ involving the Smith-Magenis chromosome region in a girl with multiple congenital anomalies. Am J Med Genet A 2006;140(1):88-91

9 Winters WD, Effmann EL. Congenital masses of the lung: prenatal and postnatal imaging evaluation. J Thorac Imaging 2001;16(4): 196-206 
16 MDS Associated with CLE Mahgoub et al.

10 Ulku R, Onat S, Ozçelik C. Congenital lobar emphysema: differential diagnosis and therapeutic approach. Pediatr Int 2008;50(5):658-661

11 Laberge JM, Puligandla P, Flageole $\mathrm{H}$. Asymptomatic congenital lung malformations. Semin Pediatr Surg 2005;14(1):16-33

12 Correia-Pinto J, Gonzaga S, Huang Y, Rottier R. Congenital lung lesions-underlying molecular mechanisms. Semin Pediatr Surg 2010;19(3):171-179
13 Konishi H, Takahashi T, Kozaki K, et al. Detailed deletion mapping suggests the involvement of a tumor suppressor gene at $17 \mathrm{p} 13.3$, distal to $\mathrm{p} 53$, in the pathogenesis of lung cancers. Oncogene 1998; 17(16):2095-2100

14 Hersh CP, DeMeo DL, Silverman EK. National Emphysema Treatment Trial state of the art: genetics of emphysema. Proc Am Thorac Soc 2008;5(4):486-493 